\title{
Selection Criteria for Word Trigger Pairs in Language Modeling
}

\author{
Christoph Tillmann and Hermann Ney \\ Lehrstuhl für Informatik VI, RWTH Aachen - University of Technology \\ D-52056 Aachen, Germany \\ \{tillmann,ney\}@informatik.rwth-aachen.de
}

\begin{abstract}
In this paper, we study selection criteria for the use of word trigger pairs in statistical language modeling. A word trigger pair is defined as a long-distance word pair. To select the most significant trigger pairs, we need suitable criteria which are the topics of this paper. We extend a baseline language model by a single word trigger pair and use the perplexity of this extended language model as selection criterion. This extension is applied to all possible trigger pairs, the number of which is the square of the vocabulary size. When using a unigram language model as baseline model, this approach produces the mutual information criterion used in $[7,11]$. The more interesting case is to use this criterion for a more powerful model such as a bigram/trigram model with a cache. We study different variants for including word trigger pairs into such a language model. This approach produced better word trigger pairs than the usual mutual information criterion. When used on the Wall Street Journal corpus, the trigger pairs selected reduced the perplexity of a full language model (trigram/cache) from 138 to 128 for a 5 million word training set and from 92 to 87 for a 38 million word training set.
\end{abstract}

\section{Introduction}

In speech recognition, the most widely used and successful language model is the so-called $N$-gram model, e. g. a bigram or trigram model, where the dependency of the word under consideration is limited to the immediate predecessor words. However it is clear that some sort of long-distance dependencies exist as well. The main goal in this paper is to include long-distance dependencies into the language model by means of so-called "trigger pairs" [7, 11]. In this work, we restrict ourselves to trigger pairs where both the triggered and the triggering events are single words (as opposed to word phrases). Unlike the approach presented in $[1,7]$, where the trigger pairs are selected on the basis of a mutual information criterion, the selection criterion presented in this paper is directly the perplexity improvement obtained by extending the baseline language model by a single trigger pair. What makes the selection criteria for word pair triggers interesting in general, is the following broader view: Given a baseline language model, how can we improve this model by including additional types of dependencies? For the selection criterion, we consider two variants. In the first variant, we directly combine trigger pairs with a given baseline model using a backing-off scheme 
[6]. When using a unigram language model as baseline model, this approach produces the mutual information criterion used by Rosenfeld in [11].

The second variant we examined is based on the idea that trigger pairs in a language model are important to the extend they can improve a given baseline model. We thus adapted the selection criterion to exploit dependencies for trigger pairs beyond what is really supplied by a given baseline model. We proved that there are such dependencies.

Section 2 covers the mathematical models of the two selection criteria presented in this paper. In Section 3 we present the main experimental results. Examples of the trigger pairs, which were computed by the different methods, are presented. The identity of these examples significantly varies for the different methods. In the last section perplexity results are presented, where a trigram model with cache is improved by trigger pairs. The perplexity improvements achieved with the trigger pairs selected by the criteria presented in $[7,11]$ were much smaller.

\section{Selection Criteria for Trigger Pairs}

The goal of this paper is to reduce the perplexity of a given baseline language model $p(w \mid h)$ by means of word trigger pairs. $p(w \mid h)$ stands for a full language model, where the word $w$ is predicted by the history $h$, which consists of the preceding words at a given position in the corpus. From the $V^{2}$ trigger pair candidates, where $V$ is the number of words in the vocabulary, those trigger pairs are selected that best improve $p(w \mid h)$. The selection criteria are in terms of the direct perplexity improvement by a trigger pair on $p(w \mid h)$. This approach to select a trigger pair to extend a given model can be compared to the so-called feature selection in [2]. We present two new selection criteria: high level trigger and low level trigger selection.

\subsection{High Level Triggers}

In order to select a trigger pair, we fix a long distance trigger pair $(a, b)$ and define an improved model $p_{a b}(w \mid h)$ :

$$
p_{a b}(w \mid h)=\left\{\begin{array}{cl}
q(b \mid a) & \text { if } a \in h \text { and } w=b \\
{[1-q(b \mid a)] \cdot \frac{p(w \mid h)}{\sum_{w^{\prime} \neq b} p\left(w^{\prime} \mid h\right)}} & \text { if } a \in h \text { and } w \neq b \\
q(b \mid \bar{a}) & \text { if } a \notin h \text { and } w=b \\
{[1-q(b \mid \bar{a})] \cdot \frac{p(w \mid h)}{\sum_{w^{\prime} \neq b} p\left(w^{\prime} \mid h\right)}} & \text { if } a \notin h \text { and } w \neq b
\end{array}\right.
$$

where $q(b \mid a)$ and $q(b \mid \bar{a})$ are two interaction parameters. Note, that for symmetry reasons we have introduced a special interaction parameter $q(b \mid \bar{a})$, when $a$ has 
not been seen in the history. The $q(b \mid a)$ and $q(b \mid \bar{a})$ are chosen to maximize the likelihood of the training corpus given the model $p_{a b}(w \mid h)$.

We now consider the difference between the log-perplexity $F_{a b}$ of $p_{a b}(w \mid h)$ and the log-perplexity $F_{0}$ of the baseline model $p(w \mid h)$ on a corpus of size $N$. The aim is to find simpler expressions to calculate $F_{a b}-F_{0}$.

$$
\begin{aligned}
F_{a b}-F_{0} & =\sum_{n=1}^{N} \log \frac{p_{a b}\left(w_{n} \mid h_{n}\right)}{p\left(w_{n} \mid h_{n}\right)} \\
& =\sum_{h}\left[N(a ; h, b) \log \frac{q(b \mid a)}{p(b \mid h)}+N(a ; h, \bar{b}) \log \frac{1-q(b \mid a)}{1-p(b \mid h)}\right. \\
& \left.+N(\bar{a} ; h, b) \log \frac{q(b \mid \bar{a})}{p(b \mid h)}+N(\bar{a} ; h, \bar{b}) \log \frac{1-q(b \mid \bar{a})}{1-p(b \mid h)}\right]
\end{aligned}
$$

where the count $N(a ; h, b)$ is to be read as: number of occurences of the word $b$ with history $h$ in the training corpus so that $h$ includes the word $a$. Multiple occurences of $a$ are counted only once. Disregarding the dependency on $h$ we define:

$$
\begin{aligned}
& N(a ; b)=\sum_{n: a \in h_{n}, b=w_{n}} 1 \\
& N(a ; \bar{b})=\sum_{n: a \in h_{n}, b \neq w_{n}} 1 \\
& N(\bar{a} ; b)=\sum_{n: a \notin h_{n}, b=w_{n}} 1 \\
& N(\bar{a} ; \bar{b})=\sum_{n: a \notin h_{n}, b \neq w_{n}} 1,
\end{aligned}
$$

where $a \in h_{n}$ means that the word $a$ occured in history of word $w_{n}$. Because of the trigger pairs being combined with the baseline model in the above backing-off scheme on the highest level, we called the trigger pairs obtained by this method high level triggers.

For implementation purposes we found it convenient to rewrite $F_{a b}-F_{0}$ as follows. As a result of the backing-off scheme in Eq. (1) we can separate the effect of one trigger pair and a baseline model. We use the counts defined in defined by the Eq. (3) to rewrite $F_{a b}-F_{0}$ as follows:

$$
\begin{aligned}
F_{a b}-F_{0} & =N(a ; b) \log q(b \mid a)+N(a ; \bar{b}) \log [1-q(b \mid a)] \\
& +N(\bar{a} ; b) \log q(b \mid \bar{a})+N(\bar{a} ; \bar{b}) \log [1-q(b \mid \bar{a})] \\
& -S(b)
\end{aligned}
$$

where $S(b)$ is:

$$
S(b)=\sum_{n=1}^{N} \delta\left(w_{n}, b\right) \log \frac{p\left(b \mid h_{n}\right)}{1-p\left(b \mid h_{n}\right)}+\sum_{n=1}^{N} \log \left[1-p\left(b \mid h_{n}\right)\right] .
$$


It is interesting to note that $S(b)$ is independent of the triggering word $a$. From this representation we draw the conclusions:

- We obtain maximum-likelihood estimates for the $q(b \mid a)$ by taking the derivates in Eq. (4) and setting the resulting equation to zero:

$$
\begin{aligned}
q(b \mid a) & =\frac{N(a, b)}{N(a, b)+N(a, \bar{b})} \\
q(b \mid \bar{a}) & =\frac{N(\bar{a}, b)}{N(\bar{a}, b)+N(\bar{a}, \bar{b})},
\end{aligned}
$$

- If we fix a triggered word $b$ and consider the triggering words $a_{i}, i=1,2, \ldots$ then the ranking of the $a_{i}$ does not depend on the identity of the baseline model.

Implementation. The real problem of computing Eq. (4) is the second term in Eq. (5). It amounts to computing a corpus perplexity for each word in the vocabulary. To manage this computational problem, we used sampling. Typically we took every 20 -th word to compute the sum $\sum_{n=1}^{N} \log \left[1-p\left(b \mid h_{n}\right)\right]$. We compared this sampling approximation with the exact calculation on a training corpus of 5 million words and found for the tested words that sampling works quite well. To calculate the perplexity improvement $F_{a b}-F_{0}$ for the high level triggers, we first compute $S(b)$ for each triggered word $b$ by using sampling. Secondly we need the trigger counts. An index structure is used, containing for each word $a$ the positions of its occurrence in the corpus. For each triggering word $a$, we have to run once through all its positions in the corpus to get all the counts we need to compute the log-perplexity $F_{a b}-F_{0}$. For the following two criteria no sampling is needed, because ...

\subsection{Unigram Triggers}

Using a unigram model $p(w)$ as baseline model $p(w \mid h)$ we get:

$$
\begin{aligned}
F_{a b}-F_{0} & =N(a ; b) \log \frac{q(b \mid a)}{p(b)}+N(a ; \bar{b}) \log \frac{1-q(b \mid a)}{1-p(b)} \\
& +N(\bar{a} ; b) \log \frac{1-q(b \mid \bar{a})}{p(b)}+N(\bar{a} ; \bar{b}) \log \frac{1-q(b \mid \bar{a})}{1-p(b)}
\end{aligned}
$$

If we multiply Eq. (8) by $1 / N$ and suppose $p(a ; b)=\frac{N(a ; b)}{N}$ we get exactly the mutual information criterion, used in $[7,11]$. Thus this criterion is simply the improvement on the log-perplexity of a unigram model by the above backing-off model for one trigger pair. The trigger pairs selected by this criterion are called unigram trigger. 


\subsection{Low Level Triggers}

Considering the model defined so far, there might be a drawback due to the following fact. The probability $q(b \mid a)$ in definition (1) is used whenever $w_{n}=b$ and $a \in h_{n}$, disregarding the probably high value of $p\left(w_{n} \mid h_{n}\right)$. The experimental results suggested another approach: To use trigger pairs only in positions where the probability $p_{S}\left(w_{n} \mid h_{n}\right)$ of a specific language model is less than a treshhold. We define an interpolated model as follows:

$$
p(w \mid h)=[1-\lambda] \cdot p_{S}(w \mid h)+\lambda \cdot \beta(w)
$$

where $\beta(w)$ is the unigram distribution of the words $w$ in the corpus. We replace $\beta(w)$ by a new distribution $\beta_{a b}(w)$, incorporating a trigger pair $a \rightarrow b$ to produce a new model $p_{a b}(w \mid h)$. For the words $w_{n}$ in positions $n$ there is actually no difference between $p_{a b}\left(w_{n} \mid h_{n}\right)$ and $p\left(w_{n} \mid h_{n}\right)$, if $p_{S}\left(w_{n} \mid h_{n}\right)$ is greater than a threshold $p_{0}$. We define:

$$
V(h)=\left\{w: p_{F}(w \mid h)>p_{0}\right\}
$$

where $p_{0}$ is a probability threshold. For the difference $F_{a b}-F_{0}$ in the loglikelihoods, we obtain the approximation:

$$
\begin{aligned}
F_{a b}-F_{0} & =\sum_{n=1}^{N} \log \frac{p_{a b}\left(w_{n} \mid h_{n}\right)}{p\left(w_{n} \mid h_{n}\right)} \\
& =\left[\sum_{n: w_{n} \notin V\left(h_{n}\right)}+\sum_{n: w_{n} \in V\left(h_{n}\right)}\right] \log \frac{p_{a b}\left(w_{n} \mid h_{n}\right)}{p\left(w_{n} \mid h_{n}\right)} \\
& \cong \sum_{n: w_{n} \notin V\left(h_{n}\right)} \log \frac{p_{a b}\left(w_{n} \mid h_{n}\right)}{p\left(w_{n} \mid h_{n}\right)} \\
& \cong \sum_{n: w_{n} \notin V\left(h_{n}\right)} \log \frac{\beta_{a b}\left(w_{n} \mid h_{n}\right)}{\beta\left(w_{n}\right)},
\end{aligned}
$$

The low level triggers are selected, using $F_{a b}-F_{0}$. Using the approximation (9) this amounts in using a reduced corpus, cosnsisting of all positions $n$, where $w_{n} \notin V\left(h_{n}\right) . \beta_{a b}$ is defined as in Eq. (1), where $p(w)$ is the unigram distribution of the reduced corpus. The trigger interaction parameters $q(b \mid a)$ and $q(b \mid \bar{a})$ are estimated on the reduced corpus, too. These trigger pairs we call low level triggers to oppose them to the high level triggers. The words $w_{n} \in V\left(h_{n}\right)$ are omitted as triggered events. But we allowed those words $w_{n}$ to trigger words following in the corpus. This was done to have efficient data to get reliable trigger counts.

\section{$3 \quad$ Experimental Results}

We computed trigger pairs for three selection criteria: 


$$
\begin{array}{lll}
\text { A: unigram selection criterion } & \text { in Eq. (8) } \\
\text { B: high level selection criterion } & \text { in Eq. (1) } \\
\text { C: low level selection criterion } & \text { in Eq. (9). }
\end{array}
$$

For the experiments we used training corpora from the Wall Street Journal task (WSJ task ) [10]. There were three different corpora of 1, 5 and 38 million words. In the first part of this section we present samples of the selected pairs for the three criteria. They were computed on the 38 million word corpus. These samples we found typical after having gone through hundreds of examples of trigger pairs. In the second part we present perplexity results on test data.

\subsection{Examples of Trigger Pairs}

Considering trigger pairs, where triggering and triggered event are single words, we generally have $V^{2}$ candidates, where $V$ is the size of the used vocabulary. Only trigger pairs that co-occured at least 3 times in a window of length 200 were used to calculated the perplexity improvement $F_{a b}-F_{0}$ according to the different criteria. For the unigram and low level triggers to carry out the calculation for all the above candidates took a maximum of 6 hours on the 38 million corpus to compute all trigger pair perplexities (on our Silicon Graphics Workstations with R 4000 processors). For the high level triggers the computation time was dominated by the need of sampling and depended on the sampling rate. We thus present for all three methods the best trigger pairs out of $V^{2}$ candidates.

As far as WSJ task mainly consists of financial texts and the trigger pairs from this domain dominate. Two tables show samples of trigger pairs obtained. Three lists of the best trigger pairs according to the three criteria are given in Table 1. For all three methods same-root triggers of the type $a \rightarrow a^{\prime} s$ and $a \rightarrow a s$, where a noun $a$ triggers its possesive $a^{\prime} s$ or its plural as, dominate. These trigger pairs have been removed, to single out the more interesting ones. Therefore the first column of Table 1 shows the position of the trigger pair within the original list. The second column presents the perplexity improvement of the extended model compared with the baseline model. The baseline model for the unigram triggers is a unigram model, for the low level and high level triggers it is a bigram model with cache. The four counts at the end of each line are the counts defined in Eq. (3).

Table 2 shows the best triggered words $b$ for a number of triggering words $a$. The words $b$ are ordered by decreasing perplexity improvement of the trigger pair $a \rightarrow b$. The trigger pairs are taken from lists of the best 500000 for each method. We now discuss the two new selection criteria in greater detail:

High Level Triggers. We found the results for the high level triggers less satisfactory than for the low level triggers, but there are some interesting facts to note with high level triggers, too. There are some trigger pairs $a \rightarrow b$, where the bigram $(b, a)$ is seen in the corpus, e.g. "Fe $\rightarrow$ Santa" The trigger pair $b \rightarrow a$ does not occur, because the corresponding word $a$ is already predicted well by 
Table 1. List of best word trigger pairs for the three selection criteria A, B and C ( self triggers and same-root triggers excluded ).

\begin{tabular}{|c|c|c|c|c|c|c|c|c|}
\hline & Rank & $\delta P P 3$ & $a$ & $b$ & $N(a, b)$ & $N(a, \bar{b})$ & $N(\bar{a}, b)$ & $N(\bar{a}, \bar{b})$ \\
\hline \multirow[t]{19}{*}{ A } & 3 & -2.22 & the & $\mathrm{a}$ & 839783 & 31263065 & 6175 & 3901023 \\
\hline & 4 & -2.21 & a & share & 15107 & 33430899 & 39833 & 2524207 \\
\hline & 5 & -1.75 & in & nineteen & 72010 & 33119066 & 54615 & 2764355 \\
\hline & 11 & -1.45 & point & dollars & 174009 & 14577007 & 66658 & 21192372 \\
\hline & 12 & -1.44 & of & the & 1793280 & 31921904 & 246783 & 2048079 \\
\hline & 13 & -1.41 & the & company & 75945 & 32026903 & 58876 & 3848322 \\
\hline & 14 & -1.29 & the & U. & 49630 & 32053218 & 47096 & 3860102 \\
\hline & 16 & -1.22 & a & the & 1985329 & 31460677 & 54734 & 2509306 \\
\hline & 17 & -1.17 & the & of & 767430 & 31335418 & 197787 & 3709411 \\
\hline & 18 & -1.10 & percent & point & 149707 & 12327082 & 92944 & 23440313 \\
\hline & 19 & -1.06 & to & be & 112112 & 33569246 & 44121 & 2284567 \\
\hline & 20 & -1.03 & the & $\mathrm{S}$. & 80343 & 32022505 & 50732 & 3856466 \\
\hline & 26 & -0.96 & the & company's & 4693 & 32098155 & 19640 & 3887558 \\
\hline & 27 & -0.95 & rose & point & 65694 & 3275140 & 176957 & 32492255 \\
\hline & 28 & -0.95 & in & the & 1778846 & 31412230 & 261217 & 2557753 \\
\hline & 29 & -0.94 & dollars & million & 128117 & 16221255 & 42062 & 19618612 \\
\hline & 32 & -0.90 & the & to & 895618 & 31207230 & 36689 & 3870509 \\
\hline & 33 & -0.89 & nine & point & 122853 & 9678103 & 119798 & 26089292 \\
\hline & 37 & -0.86 & dollars & cents & 53792 & 16295580 & 4828 & 19655846 \\
\hline \multirow[t]{20}{*}{$\mathrm{B}$} & 18 & -0.0103 & Texaco & Pennzoil & 1423 & 294204 & 433 & 35713986 \\
\hline & 19 & -0.0102 & Pennzoil & Texaco & 1911 & 152412 & 2312 & 35853411 \\
\hline & 30 & -0.0074 & $\mathrm{Fe}$ & Santa & 1111 & 95276 & 1379 & 35912280 \\
\hline & 34 & -0.0071 & distillers & Guinness & 835 & 79004 & 802 & 35929405 \\
\hline & 38 & -0.0064 & $\mathrm{Am}$ & Pan & 1241 & 346056 & 975 & 35661774 \\
\hline & 41 & -0.0062 & Campeau & Federated & 844 & 134468 & 542 & 35874192 \\
\hline & 45 & -0.0061 & Cola & Coca & 807 & 144817 & 634 & 35863788 \\
\hline & 64 & -0.0051 & oil & Opec & 2274 & 2138246 & 221 & 33869305 \\
\hline & 72 & -0.0048 & Federated & Campeau & 941 & 129385 & 856 & 35878864 \\
\hline & 107 & -0.0039 & multip & negot & 367 & 54612 & 86 & 35954981 \\
\hline & 130 & -0.0035 & Geller & Lord & 494 & 26838 & 652 & 35982062 \\
\hline & 131 & -0.0035 & Beazer & Koppers & 262 & 25132 & 131 & 35984521 \\
\hline & 137 & -0.0034 & soviet & Moscow & 1712 & 1173777 & 663 & 34833894 \\
\hline & 163 & -0.0031 & rales & Interco & 243 & 22795 & 147 & 35986861 \\
\hline & 165 & -0.0031 & Eddie & crazy & 478 & 67269 & 565 & 35941734 \\
\hline & 171 & -0.0030 & Arabia & Saudi & 802 & 147960 & 1145 & 35860139 \\
\hline & 181 & -0.0029 & Warner & Borg & 345 & 204029 & 132 & 35805540 \\
\hline & 182 & -0.0029 & Shield & Robins & 731 & 104266 & 517 & 35904532 \\
\hline & 190 & -0.0028 & Robins & Dalkon & 295 & 80880 & 40 & 35928831 \\
\hline & 192 & -0.0028 & Shoreham & Lilco & 247 & 29555 & 146 & 35980098 \\
\hline \multirow[t]{19}{*}{$\bar{C}$} & 1 & -0.00371 & neither & nor & 411 & 28775 & 567 & 1853529 \\
\hline & 14 & -0.00109 & tip & iceber & 55 & 4944 & 4 & 1878279 \\
\hline & 15 & -0.00107 & soviet & Moscow's & 119 & 80652 & 26 & 1802485 \\
\hline & 26 & -0.00101 & named & $\mathrm{s}$ & 147 & 63692 & 164 & 1819279 \\
\hline & 27 & -0.00100 & Iraq & Baghdad & 74 & 13766 & 45 & 1869397 \\
\hline & 33 & -0.00093 & Eastm & Kod: & 49 & 3919 & 16 & 1879298 \\
\hline & 40 & -0.00090 & Eastman & photographic & 55 & 3913 & 61 & 1879253 \\
\hline & 43 & -0.00089 & Carbide & Danbury & 51 & 3350 & 46 & 1879835 \\
\hline & 50 & -0.00088 & Eurodollar & syndication & 60 & 3758 & 139 & 1879325 \\
\hline & 55 & -0.00086 & filed & alleges & 103 & 52441 & 80 & 1830658 \\
\hline & 57 & -0.00085 & asked & replied & 120 & 67419 & 110 & 1815633 \\
\hline & 60 & -0.00085 & Kodak & photographic & 57 & 6367 & 59 & 1876799 \\
\hline & 68 & -0.00083 & motor & Ford's & 74 & 25221 & 47 & 1857940 \\
\hline & 71 & -0.00083 & South & Pretoria & 87 & 71047 & 18 & 1812130 \\
\hline & 75 & -0.00080 & Iran & Baghdad & 80 & 42050 & 39 & 1841113 \\
\hline & 76 & -0.00080 & occupational & Osha & 40 & 3011 & 12 & 1880219 \\
\hline & 80 & -0.00079 & soviet & Moscow & 100 & 80671 & 45 & 1802466 \\
\hline & 81 & -0.00079 & machines & Armonk & 68 & 29004 & 28 & 1854182 \\
\hline & 86 & -0.00077 & Peabody & Kidder's & 49 & 8388 & 22 & 1874823 \\
\hline
\end{tabular}


Table 2. List of best triggered words $b$ for some triggering words $a$ for the selection criteria $\mathrm{A}, \mathrm{B}$ and $\mathrm{C}$.

\begin{tabular}{|c|c|}
\hline$a$ & $\bar{b}$ \\
\hline asked & $\begin{array}{l}\text { A: point replied Mr. I he percent asked one seven eight } \\
\text { B: Deltona Prism Benequity Taiyo Ropak Genesis Quintessential Envirodyne target's Teamster } \\
\text { C: replied answered responded refused replies responses reply yes request requesting }\end{array}$ \\
\hline airlines & $\begin{array}{l}\text { A: airlines airline air passenger fares carriers traffic flights miles continental } \\
\text { B: Delta's Northwest's Maxsaver Transtar Swissair Primark United's Motown Airbus's Cathay } \\
\text { C: American's passengers Airlines' Eastern's United's hubs fares Northwest's carriers flights }\end{array}$ \\
\hline buy & $\begin{array}{l}\text { A: buy shares stock dollars company price offer million share stake } \\
\text { B: Sheller Deltona Motown Northview Barren Philipp Selkirk Oshkosh Radnor Bumble } \\
\text { C: repurchased Landover purchases repurchases Kohlberg repurchase Southland's undervalued }\end{array}$ \\
\hline concerto & $\begin{array}{l}\text { A: orchestra concerto music symphony piano violin philharmonic ballet composer concert } \\
\text { B: Mozart violin Bach poignant } \\
\text { C: strings orchestra violin score Mozart pianist recordings keyboard listen variations }\end{array}$ \\
\hline Ford & $\begin{array}{l}\text { A: Ford Ford's cars auto Chrysler car G. Jaguar models M. } \\
\text { B: Ford Ford's Edsel ambulances Dearborn Jaguar Bronco Mustang Jaguar's Sheller } \\
\text { C: Ford's Dearborn Bronco Taurus Escort Chrysler's Tempo Mustang Thunderbird subcompact }\end{array}$ \\
\hline love & $\begin{array}{l}\text { A: her love she point his I said dollars percent You } \\
\text { B: Genex polly soothing boyish pathetic authenticity quaint Horace chalk Domino's } \\
\text { C: beautifully passion sweet sexy romantic hero pop lovers pale wit }\end{array}$ \\
\hline Microsoft & $\begin{array}{l}\text { A: Microsoft software Lotus computer Microsoft's Apple computers personal O. one } \\
\text { B: Microsoft Microsoft's Borland Ashton Lotus's Adobe Oracle Redmond Novell Bausch } \\
\text { C: Microsoft's Redmond Apple's Borland spreadsheets Ashton Lotus's database spreadsheet }\end{array}$ \\
\hline says & $\begin{array}{l}\text { A: says said point million dollars adds seven he five one } \\
\text { B: Benham Barren accredited Philipp Panasonic Radnor Deltona kids' Battelle Motown } \\
\text { C: concedes explains adds agrees recalls asks insists acknowledges asserts predicts }\end{array}$ \\
\hline
\end{tabular}

the bigram model. In Table 1 the high level triggers only consist of proper names. Looking at the text all of them seem reasonable within the domain of Wall Street Journal business texts. Table 2 shows that the high level method fails to produce meaningful trigger pairs in some cases. An interesting fact to notice with high level triggers is that only 3000 out of $V^{2}$ possible trigger pairs were able to improve a given bigram model with cache. This is because the current word is predicted by a trigger pair with no regard to whether it is already predicted well by the bigram model with cache or not. From all this we draw the conclusion that trigger effects in general tend to be too weak to improve on a full baseline model in a backing-off fashion presented in this paper and that one should prefer a scheme, where a choice is made for when to use trigger pairs. This is done with the low level trigger pairs as introduced in this paper.

Low Level Triggers. In both tables the low level trigger pairs yield the best results in most cases. To understand them you sometimes have to take a close look at the underlying corpus, consisting of business texts. Some words produce very interesting trigger pairs, e.g. the verbs "asked" and "says" that mostly trigger verbs again, which even agree with them in tense. Another interesting example are the nouns "airlines" and "Ford", where the corresponding low level triggers show names of airlines or names of car models build by "Ford". The corresponding unigram triggers look worse for verbs, but for some nouns they 
seem to have a kind of generalization capability in some cases.

The low level triggers resulted from using counts from a reduced corpus. It consisted of all positions of a given corpus of 38 million words for which a baseline model $p(w \mid h)$ computed a probability less than a given threshold $p_{0}=0.8 * 1 / V$, where $V$ is the number of words in the vocabulary. The baseline model was a bigram model trained on the same corpus, which was interpolated with a cache component with a weight of 0.1 . Using that threshold 1.8 million positions were left, where the actual history $h$ did not provide sufficient information with the baseline model $p(w \mid h)$ for the actual word $w$ and where we want to rely on trigger pairs. We used different thresholds, but changing them has only a small effect on the selection of the calculated trigger pairs or the perplexity results. We emphasize the following facts with low level triggers:

- Among the best low level triggers are nouns that trigger their possessives, while self triggers do not occur at all.

- As well as using a probability threshold the corpus could be reduced by using only corpus positions $n$ where the corresponding bigram $\left(w_{n}, w_{n-1}\right)$ was seen only once and where $w_{n}$ was not contained in the history $h_{n}$. The resulting pairs look very much the same.

- If we confine the history to the current sentence, we get trigger pairs, showing more grammatical structure, e.g. "I $\rightarrow$ myself", "We $\rightarrow$ ourselves". These results can be compared to the link grammar results in [4], where the grammar consists simply of pair of words.

The choice of pairs being used to extend a full language model depends on the model to be extended. The unigram trigger might offer a greater average usefulness in terms of mutual information, but the low level triggers have been selected to improve a full language model, consisting of bigram and cache. The perplexity results prove that they manage to provide information that supplements the information by bigram and cache.

\section{$3.2 \quad$ Perplexity Results}

In this subsection we present perplexity results which were achieved with the calculated trigger pairs on a trigram model with cache. We used the following model to incorporate the selected trigger pairs into a full language model:

$$
p\left(w_{n} \mid h_{n}\right)=\left(1-\lambda_{1}-\lambda_{2}\right) \cdot p_{S}\left(w_{n} \mid h_{n}\right)+\lambda_{1} \cdot p_{C}\left(w_{n} \mid h_{n}\right)+\lambda_{2} \cdot p_{T}\left(w_{n} \mid h_{n}\right) \quad .
$$

where the history $h_{n}=w_{n-M}^{n-1}$ consists of the $M$ predeccesor words of $w_{n}$. The cache probability $p_{C}\left(w_{n} \mid w_{n-M}^{n-1}\right)$ is defined as:

$$
p_{C}\left(w_{n} \mid w_{n-M}^{n-1}\right)=\frac{1}{M} \sum_{m=1}^{M} \delta\left(w_{n} \mid w_{n-m}\right)
$$


with $\delta(w, v)=1$ if and only if $w=v$. The trigger model is defined as:

$$
p_{T}\left(w_{n} \mid w_{n-M}^{n-1}\right)=\frac{1}{M} \sum_{m=1}^{M} \alpha\left(w_{n} \mid w_{n-m}\right) .
$$

The $\alpha(b \mid a)$ are obtained by renormalization:

$$
\alpha(b \mid a)=\frac{q(b \mid a)}{\sum_{b^{\prime}} q\left(b^{\prime} \mid a\right)},
$$

where the $q(b \mid a)$ are the maximum likelihood estimates as defined in Eq. (7). This renormalization is due to the fact that not all computed trigger pairs are used in a trigger model. In the experiments the history $h$ consisted of all those words starting from the last article delimiter.

Perplexities were computed using a corpus of 325000 words from the WSJ task. We used the computed word pairs together with a cache in an interpolated model. The $\lambda_{i}$ in Eq. (10) were adjusted by trial and error in informal experiments. They can be trained by the EM procedure $[3,5]$. The baseline trigram model was a backing-off model presented in [9]. We choose a number of the best trigger pairs as judged by the different selection criteria. We suppose that the combination of these trigger pairs will yield the best perplexity improvement within the model defined in Eq. (10). The problem with all the selection criteria presented is that the combination of the selected trigger pairs into one global language model is not captured by any of the criteria. However the low level criterion provides a better approximation to the use of the trigger pairs in Eq. (10). As opposed to the low level triggers, the high level triggers were not able to achieve perplexity improvements because the model defined in Eq. (10) is inadequate. In a first simple experiment we try to improve on a unigram model with

Table 3. Perplexity results for a unigram language model ( 5 million training words) with triggers and cache.

\begin{tabular}{|l|r|}
\hline model & 5 Mio \\
\hline unigram & 1027 \\
\hline + low level triggers & 960 \\
+ unigram triggers & 860 \\
\hline + cache & 750 \\
\hline
\end{tabular}

the unigram triggers and the low level triggers in Table 3 . The unigran model was trained on the 5 million corpus. We used the 500000 best trigger pairs for low level and unigram triggers. The unigram triggers improve on an that unigram model to a much higher extend than the low level triggers can do. This is because the unigram triggers were selected to improve on an unigram model, whereas the low level triggers were selected to improve on a trigram model with 
Table 4. Perplexity results for a trigram language model ( 1,5 and 38 million trainings words ) with triggers and cache.

\begin{tabular}{|l|r|r|r|r|}
\hline model & Number of Pairs & 1 Mio & 5 Mio & 38 Mio \\
\hline trigram with no cache & & 252 & 168 & 105 \\
\hline trigram/cache & & 197 & 138 & 92 \\
\hline + unigram triggers & 1500000 & 191 & 135 & 91 \\
+ low level triggers & 500000 & 182 & 130 & 88 \\
+ low level triggers & 1500000 & 180 & 128 & 87 \\
\hline
\end{tabular}

cache. On the other hand the low level triggers were capable of improving on a trigram model with cache, which could not be achieved by using the original unigram triggers as shown in Table 4 . The experiments with the trigram language model were carried out for different numbers of trigger pairs. The second column shows the number of the used trigger pairs. Using unigram triggers we weren't capable of achieving the same improvements as with the low level triggers.

The best results were obtained by employing the best 1.5 million trigger pairs. They prove that the low level triggers improve the trigram model with cache. Using 500000 instead of 1500000 low level triggers only slightly changes the results.

\section{Summary}

In this paper, we considered the problem of selecting trigger pair pairs for language modeling. Rather than using some more or less arbitrary selection criterion, we presented a new method for finding word trigger pairs: given a reference language model to start with, we extend it by including a word trigger pair and compute the perplexity improvement of this extended model over the reference model. This perplexity improvement is used as selection criterion. For the special case of a unigram reference model, this new method is identical with the mutual information criterion. In the experimental tests, we found that the new method produces better results:

1. The selection criterion for the low level triggers produces intuitively better word trigger pairs than the usual mutual information criterion.

2. When used in a full language model, consisting of trigram model and cache the introduced low level triggers reduce the perplexity from 138 to 128 for the 5 -million training set and from 92 to 87 for the 38 -million training set. In comparison, when using the conventional mutual information criterion, the perplexity improvements were significantly smaller.

\section{References}

1. L.R. Bahl, F. Jelinek, R.L. Mercer and A. Nadas. "Next Word Statistical Predictor". IBM Techn. Disclosure Bulletin, 27(7A), pp. 3941-3942, 1984. 
2. A. Berger, S. Della Pietra and V. Della Pietra. "A Maximum Entropy Approach to Natural Language Processing". In Computational Linguistics, Vol. 22, No. 1, pp. 39-71, March 1996.

3. A.P. Dempster, N.M. Laird and D.B. Rubin. "Maximum Likelihood from Incomplete Data via the EM Algorithm". In Journal of the Royal Statistical Society, Vol. 39, No. 1, pp. 1-38, 1977.

4. S. Della Pietra, V. Della Pietra, J. Gillett, J. Lafferty, H. Printz and L. Ures. "Inference and Estimation of a Long-Range Trigram Model". In Lecture Notes in Artificial Intelligence, Grammatical Inference and Applications, ICGI-94, Alicante, Spain, Springer-Verlag, pp. 78-92, September 1994.

5. F. Jelinek. "Self-Organized Language Modeling for Speech Recognition". In Readings in Speech Recognition, A. Waibel and K.F. Lee (eds.), pp. 450-506, MorganKaufmann, 1991.

6. S.M. Katz. "Estimation of Probabilities from Sparse Data for the Language Model Component of a Speech Recognizer". In IEEE Trans. on Acoustics, Speech and Signal Processing, Vol. 35, pp. 400-401, March 1987.

7. R. Lau, R. Rosenfeld and S. Roukos. "Trigger-Based Language Models: A Maximum Entropy Approach". In Proc. IEEE Int. Conf. on Acoustics, Speech and Signal Processing, Minnesota, MN, pp. II 45-48, April 1993.

8. R. Lau, R. Rosenfeld and S. Roukos. "Adaptive Language Modeling Using the Maximum Entropy Approach". In Proceedings of the ARPA Human Language Technology Workshop, pp. 108-113, Morgan-Kaufmann, March 1993.

9. H. Ney, M. Generet and F. Wessel. "Extensions of Absolute Discounting for Language Modeling". In Fourth European Conference on Speech Communication and Technology, pp. 1245-1248, Madrid, September 1995.

10. D.B. Paul and J.B. Baker. "The Design for the Wall Street Journal-based CSR Corpus". In Proceedings of the DARPA SLS Workshop, pp. 357-361, February 1992.

11. R. Rosenfeld. "Adaptive Statistical Language Modeling: A Maximum Entropy Approach". Ph.D. thesis, School of Computer Science, Carnegie Mellon University, Pittsburgh, PA, CMU-CS-94-138, 1994.

This article was processed using the $\mathrm{AAT}_{\mathrm{E}} \mathrm{X}$ macro package with LLNCS style 